\title{
WI-MOB: Power Transmission System for Charging Smart Phones using RF Module through Wi-Fi
}

\author{
Shareen Noronha ${ }^{1}$, Prajna $\mathrm{P}^{1}$, Prateeksha Prasad ${ }^{1}$, Pratheeksha $^{1}$, Saritha ${ }^{1}$, Sachin Bhat ${ }^{1}$ \\ Dept. of ECE, SMVITM, Udupi, Karnataka ${ }^{1}$
}

\begin{abstract}
Wireless technology has shown an advance improvement and is rapidly growing making the life of mankind much easier and feasible. The Wi-Fi plays a major role in the wireless power transmission in this paper. The range of a $\mathrm{Wi}-\mathrm{Fi}$ is wider compared to other wireless transmitters such as Bluetooth. WI-MOB uses ESP8266 module which is a combination of antenna, voltage regulator and filters. This module is followed by an amplifier which is used to boost the signal in order to meet the required range of voltage. Wi-Fi signal frequency varies between $2.4 \mathrm{GHz}$ or $5 \mathrm{GHz}$ which is detected and captured by the PCB antenna being used. The received signal does not meet our expected values hence we amplify the signal using a boost circuit and then connect it to the cell phone. The Cell phone starts charging when it is paired with the available Wi-Fi router in the vicinity.
\end{abstract}

Keywords: RF module, PCB antenna, Voltage regulator, WiFi.

\section{INTRODUCTION}

Wireless Charging has been a boon to mankind. Charging our phones without the need for socket is what we expect these days. Dragging the charger to the required places is not possible or feasible. Wireless charging, also known as Wireless Power Transmission is one of the ways to eliminate the charging through sockets. Wireless Transmission of power gives way to use the phone without any cumbersome while charging. Wi-Fi covers a range of 150 feet indoors and 300 feet outdoors, this wide range of signal transmission enables the charging of cell phone eliminating the usage of wired chargers. These Wireless Power Transmitters enable the user to charge the phone whenever the Wi-Fi signal is available. Hence the user can use the signal to charge the phone and can use the internet too. This multi-functioning does not bother the charging process nor the usage of internet.

Compared to charging with cords, wireless charging has the following benefits:

1) Power saving method, elimination of the cord results in non-usage of the sockets and hence not much power is consumed.

2) Easy to access the open network Wi-Fi signal especially in public places such as Shopping Malls, Airports etc.

3) When the battery of the cell phone is low and needs to be charged immediately, then if there is a Wi-Fi signal in the vicinity it can be used to charge the cell phone without any need for the socket to plug-in the cord.

The Advancement in the field of wireless technology has been adverse over the years; the access to the access to the internet has become easier and feasible. The usage of Wi-Fi is wide enough and the charging of cell phone through WiFi has made the work easier. Wireless charging holds a good future when it is about producing enough amount of power to charge the mobile phones.

\section{LITERATURE SURVEY}

In order to build wireless charging prototype, it is important to study the wireless charging technology, fundamentals and applications. We have used RF radiation technology and far field charging systems proposed in this paper [1]. ESP8266 Wi-Fi module contains PCB antenna. Small size PCB antenna at high frequency is cost effective, easy to implement and performs better with more than $868 \mathrm{MHz}$. It uses mean during connecting strip which is optimized to have frequency band operation, between 2.4 to $2.49 \mathrm{GHz}$ [2]. A technique which helps in charging low power consumption devices resulting benefits in better product design, usability, and reliability is explained. The main aim is to obtain the free energy in the Wi-Fi signals which would rather go unnoticed and maintain an eco-friendly environment [3]. To improve the existing wireless power transmission system comparative study on previous systems is essential. Mainly it explains Non-Radiative, Radiative Electromagnetic induction and Electrostatic Induction [4]. Several significant improvements to the earlier design including a newly developed, wideband antenna array, rectifier, boost converter, and a modified charger circuit are proposed [5]. The performance of energy harvesting in an efficient 
way is proposed [6] by using simple voltage doubler. It involves receiving the RF signals from the Bluetooth of any device which can be further extended to Wi-Fi enabled devices. This paper [7] focuses on short distance charging method: as Bluetooth. Power charging is based on schottky diode with a real commercial film-type antenna on mobile terminal results in smart charging. It is difficult to charge smart phone battery completely. [8] Faraday's law of electromagnetic induction is the basic principle to charge the mobile phone which is proposed in this paper.

\section{MeTHOdOLOGY}

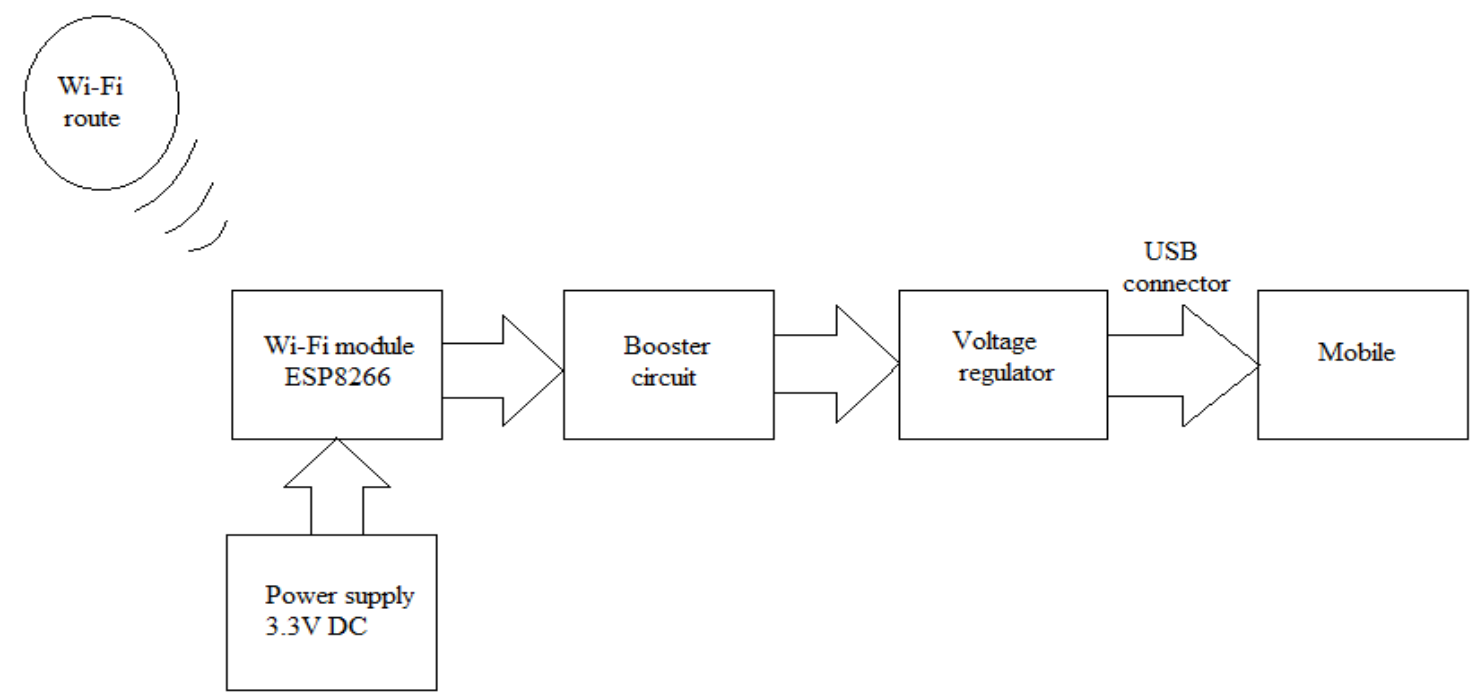

Fig.3.1 Extraction of energy from Wi-Fi

The methodology used for extracting the energy from the Wi-Fi is clearly shown in the Fig 3.1 and 3.2. It mainly involves the Wi-Fi module ESP8266 which is used to connect to the particular Wi-Fi network. This device works with 3.3V. It is a low cost Wi-Fi chip, produced by Shanghai-based Chinese manufacturer, Espressif Systems. It allows microcontroller to connect to the Wi-fi network.

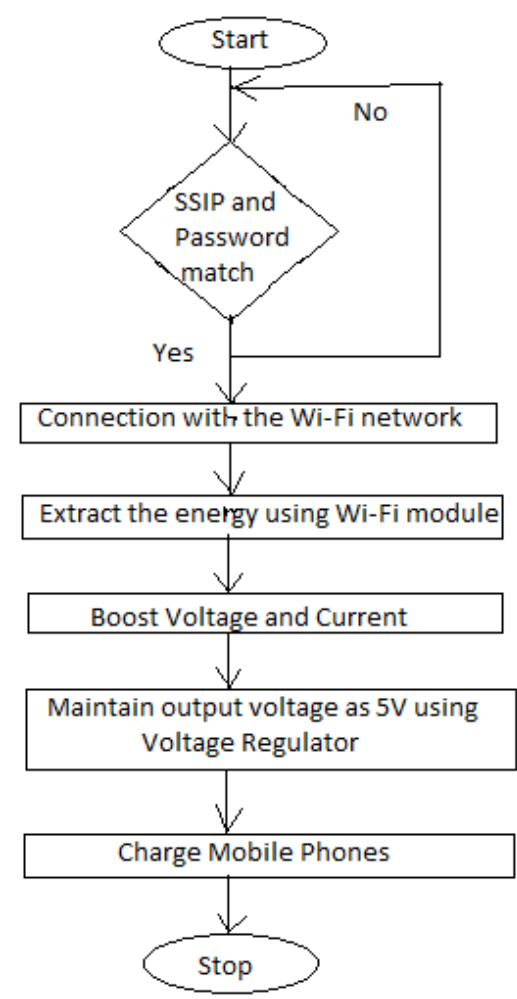

Fig. 3.2. Flow chart 
It can also make simple TCP/IP connections using Hayes-style commands. The main features of ESP8266 Wi-Fi module include 32-bit RISC CPU, instruction RAM of 64KB, data RAM of 96KB,16 GPIO pins, 10-bit ADC and WEP or WPA/WPA2 authentication.

It also involves boost circuits in which the regulated output voltage that is higher that the input voltage is generated. Here in boost circuit we are using LM2577 IC from National Semiconductor. This IC has a very wide input voltage range that is from $3.5 \mathrm{~V}-40 \mathrm{~V}$ with different output voltage versions that is $12 \mathrm{~V}, 15 \mathrm{~V}$ and adjustable. The Fig.3.2 shows the schematic of the booster circuit which we are using in our model. It is simple and also easy to build. It is cost effective. From a $5 \mathrm{~V}$ unregulated supply it is able to produce $12 \mathrm{~V}$, and the maximum output current of $800 \mathrm{~mA}$. The requirement of external components is minimum in this. It contains $52 \mathrm{kHz}$ internal oscillator. The soft-start function of this reduces In-rush currents especially during the start-up. One can find its typical applications in simple boost regulator, flyback as well as forward regulators and multiple-output regulators.

Obtained output of the booster circuit is applied to the voltage regulator. It is actually designed to maintain constant voltage level automatically. Here it is used to regulate one or more AC or DC voltages depending on the design. The output of voltage regulator is introduced to mobile through USB connector. Here the obtained voltage and current is converted in order to meet the specifications of mobile charging.

\section{A. Extracting energy from Wi-Fi signal}

The prototype contains ESP8266 Wi-Fi module. This module is mainly used to connect to a particular Wi-Fi network using its IP address. Once the network is paired, it will receive the Wi-Fi signal which is of the range $2.4 \mathrm{GHz}$. It also enables the security for accessing the Wi-Fi signal.

ESP8266 is a Wi-Fi module consists of antenna switches, RF, power amplifiers, low noise receive amplifier, filters, power management module. It requires minimal external circuitry. The module is used in two ways either for hosting an application or as offloading Wi-Fi networking functions. It requires 3.3 $\mathrm{V}$ supply to turn on the IC. It supports Wi-Fi which has the frequency range of 2.4GHz. ESP8266 finds its main application in wearable electronics and Internet of things.

\section{B. Boosting the signal}

The voltage and current obtained at the output of the Wi-Fi module is less. To meet the specification, the signal has to be boosted. The booster circuit used is shown in Fig 3.3. The circuit is designed such that the output voltage and current is $5 \mathrm{~V}$ and $0.5 \mathrm{~A}$ respectively.

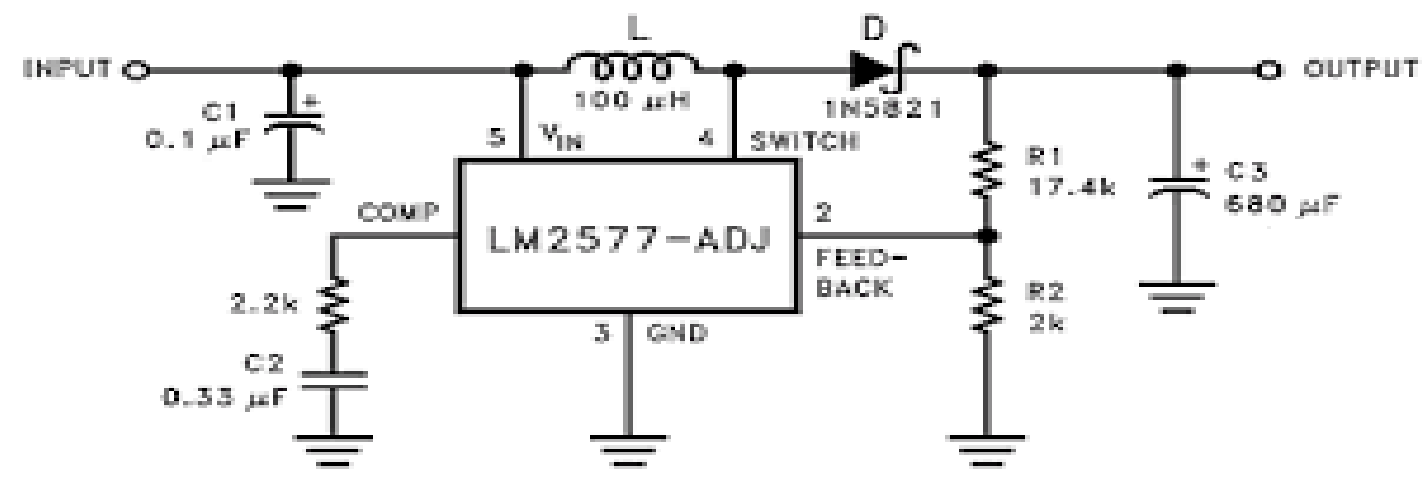

Fig 3.3 Boost Circuit

\section{Regulating the output voltage}

There is a chance of getting voltage more than $5 \mathrm{~V}$ which may cause damage to the mobile. So we require voltage regulator. Here 7805 voltage regulator IC is used, which maintains the output voltage as $5 \mathrm{~V}$. This output is used to charge the mobile.

\section{IV.RESULTS}

Fig 4.1 describes the charging of the phone through WiFi signal. The WiFi module captures the WiFi signal and transmits the required output. The output is then passed on to the booster from which the required amount of charge is obtained. The module pairs with the WiFi signals and the charging starts. 


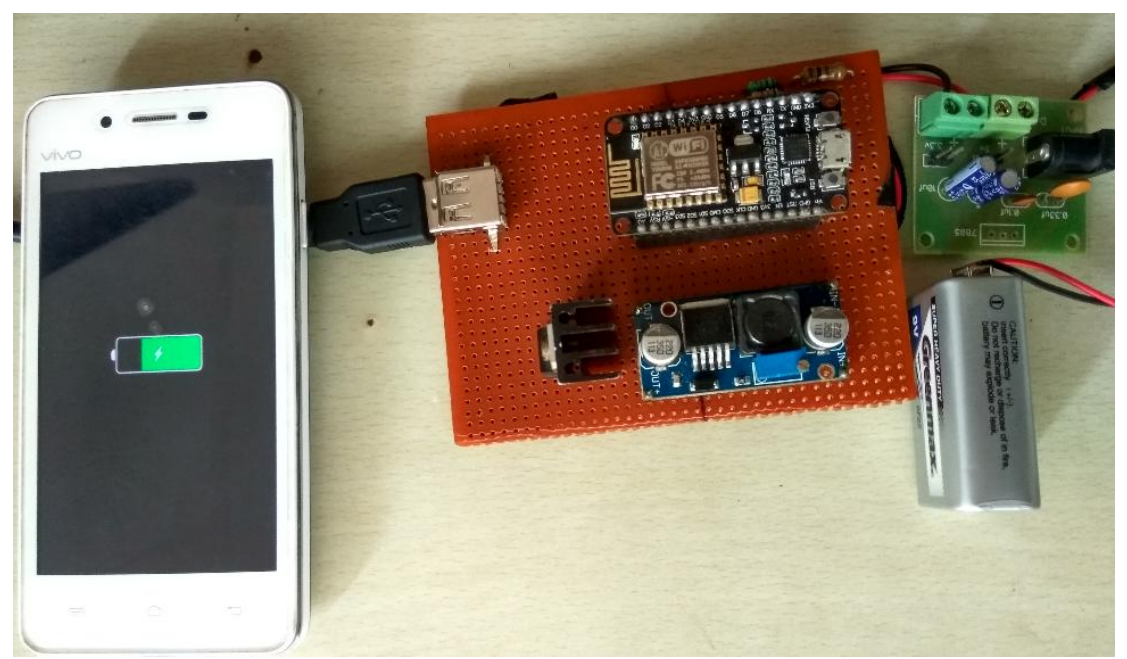

Fig 4.1. Wireless charging using WiFi

\section{CONCLUSION}

There is increasing interest in the internet of things where small computing sensors and mobile devices are embedded in everyday objects and environments. A key issue is how to power these devices as they become smaller and more numerous; plugging them into provided power is inconvenient and is difficult at a large scale. The energy harvester would be an affective solution for changing the smartphones which consumes lot of power where current is already in demand. We proposed a far field power delivery system using WiFi module. Our proposed circuit which harvest energy from WiFi signal by connecting to that particular WiFi network. As on whole the system can conveniently charge a battery of the mobile phones without the need for main supply or charging. Thus our proposal aims that a smart environment is created utilising the resources efficiently.

\section{REFERENCES}

[1] Xiao Lu, Ping Wang, Dusit Niyato, Ding Kim, Zhu Han, "Wireless Charging Technologies: Fundamentals, Standards, and Network Application". IEEE. pp. 1-40, November 2015.

[2] Sham Nayse, Mohammad Atique "A Design of PCB Antenna for Application Based Wireless Sensor Node", International Jounal of Innovative Research in Computer and Communication Engineering.Vol.2 Issue 6, pp. 4816-4821, June 2014.

[3] G.Geethanjali, K.Aparna, "A Socket-Free Charging Technique By Utilizing The Wi-Fi Signals" IOSR Journal of Computer Engineering (ISOR-JCE). pp 01-05.

[4] Priyanka Sinha, Ritesh Diwan. "Review Paper on Wireless Power Transmission for Energy Harvesting System". International Journal of Science and Research. Vol.5, Issue 5, pp. 181-186. May 2016.

[5] Kenneth Gudan, Shuai Shao, Jonathan J. Hull, Joshua Ensworth, Matthew S. Reynolds "Ultra Low Power 2.4GHz RF Energy Harvesting and Storage System with -25dBm Sensitivity". IEEE RFID ,pp 1-7, April 2015

[6] Ajay S. Sivaramakrishnan, Kailarajan Jeyaprakash Jegadishkumar. "A Highly Efficient Power Management System for Charging Mobile Phones using RF Energy Harvesting" International Journal of Information Technology Convergence and Services. Vol.1, No.5, pp. 21-30. October 2011.

[7] J. Gobinath, M. Vinoth Kumar, M. Lenin. "Short Range Wireless Charging system for Smart Phone through New Energy Harvesting Circuit". International Journal of Computer application. pp. 1-5.

[8] Tarique Salat, Shilpak Raich, Supriya Mahto, Shilpa Togarwar, "A Wireless Battery Charger for Mobile Device”. International Journal of Emerging Trends and Technology in Computer Science [Online]. Vol.2, Issue 3. pp. 103-105, May-Jun 2013 